\title{
Inverse Sturm-Liouville problems: some recent developments
}

\author{
Alan L. Andrew ${ }^{1}$
}

(Received 21 January 2011; revised 14 June 2011)

\begin{abstract}
We consider numerical methods for obtaining, from spectral data, information on the potentials of Sturm-Liouville operators. In particular, we describe some recent work on methods using an asymptotic correction technique of Paine, de Hoog and Anderssen. Topics covered include a discussion of difficulties arising from the scarcity of accurate data in physical applications, a preview of some work in progress on the Hochstadt-Lieberman problem, and suggestions for future work.
\end{abstract}

\section{Contents}

1 Introduction

C288

2 Asymptotic correction

C289

3 The Hochstadt-Lieberman problem

C291

http://anziamj.austms.org.au/ojs/index.php/ANZIAMJ/article/view/3888 gives this article, (C) Austral. Mathematical Soc. 2011. Published July 1, 2011. ISSN 1446-8735. (Print two pages per sheet of paper.) Copies of this article must not be made otherwise available on the internet; instead link directly to this URL for this article. 


\section{Introduction}

Inverse eigenvalue problems arise when we seek information about a vibrating system from observations of its normal modes of vibration. Most work on the numerical solution of these inverse problems concerns the finite dimensional case, when, for some $n \in \mathbb{N}$, we have to solve $n$ simultaneous nonlinear equations in $n$ unknowns [10]. This is more difficult than the corresponding 'direct' problem (finding all eigenvalues of a given matrix) which is equivalent to solving a single nonlinear equation - the characteristic equation. Inverse eigenvalue problems for differential equations are more difficult still, as these are infinite dimensional problems. In theory, infinitely many data points are required for their solution, and the question of which infinite sets of data will determine the solution uniquely is highly nontrivial. The theoretical literature is largely concerned with finding which infinite sets of data will suffice. This still does not address the greatest difficulty in obtaining solutions to real world problems: the severe limitations on the amount of data available. Since only a finite set of data is available in applications, standard numerical methods, including all those considered here, effectively model the problem as some finite dimensional problem $[2,21]$. Neher [23] gave a method for computing rigorous two sided bounds for the solution of one such model problem, but, with only a finite set of data, we cannot tell how close the solutions of the model problem are to those of the original inverse Sturm-Liouville problem. Different numerical methods correspond to different models, and hence yield different information about the original problem. Since it is generally lack of data rather than limited availability of computing resources that constrains the reliability of any conclusions drawn from the available data, the best strategy is often to make several independent calculations using different methods. For this reason, it is better to have a large number of available numerical methods rather than seeking to find a single 'optimal' method. 
We consider how we may use a subset of the eigenvalues $\lambda$ of the SturmLiouville problem

$$
\begin{aligned}
& -y^{\prime \prime}+q y=\lambda y, \\
& \sin \left(c_{1}\right) y(0)+\cos \left(c_{1}\right) y^{\prime}(0)=\sin \left(c_{2}\right) y(\pi)+\cos \left(c_{2}\right) y^{\prime}(\pi)=0,
\end{aligned}
$$

to estimate the potential $\mathbf{q}:[0, \pi] \rightarrow \mathbb{R}$ in (1). Even knowledge of all the (infinitely many) eigenvalues of (1) and (2) is generally insufficient to determine $q$ uniquely. For example, if $\sin \left(c_{1}+c_{2}\right)=0$ then the eigenvalues are the same for $\mathbf{q}$ as for $\widehat{\mathbf{q}}$, where $\widehat{\mathbf{q}}(x)=\mathrm{q}(\pi-x)$. For uniqueness, we generally need an additional infinite set of data. Famous sets of sufficient conditions to ensure the uniqueness of $q \in \mathrm{L}^{1}(0, \pi)$, include those of (i) the 'symmetric' problem [2], in which we are given the eigenvalues of (1) and (2), together with the symmetry requirement that $\mathrm{q}=\widehat{\mathrm{q}}$, and (ii) the closely related [4] 'two spectra' problem in which we are given the eigenvalues of (1) and (2), together with the eigenvalues of (1) with boundary conditions which coincide with (2) at one boundary but not at the other. Most other famous sets of sufficient conditions $[2,5,9,14,15,16,27,31]$ include some information about the eigenfunctions of (1) and (2). Section 3 considers an important exception - the 'Hochstadt-Lieberman' problem, where $\mathrm{q}$ is known on some subinterval of $(0, \pi)$. We announce some new results whose proofs will appear elsewhere [5].

\section{Asymptotic correction}

Several very different methods are available for the numerical solution of inverse Sturm-Liouville problems [2, 9, 14, 15, 18, 23, 25, 29, 30, 31, 32]. For example, some involve transformation of the problem to one involving integral equations $[15,31]$, and some require solution of the direct problem for a sequence of approximations to $\mathbf{q}[30,32]$. This article is primarily concerned with methods using a simple technique [6, 26] which I call 'asymptotic correction' (sometimes called 'algebraic correction' [11] or 'the AAdHP 
correction' [28]). This enables finite difference and finite element methods, which are well developed for direct problems, to be used successfully for inverse eigenvalue problems for ordinary and partial differential equations. One reason why this method is important is that it is potentially applicable to many other differential equations [3].

As discussed elsewhere [2, 3, 4, 5, 22, 24], if we simply use the eigenvalues of the differential equation as input data in the inverse problem for the corresponding finite difference or finite element matrix, then many methods for solving the matrix inverse eigenvalue problem will not work at all, and none will give satisfactory results. This is because the discrete (finite difference or finite element) eigenvalues do not have the same asymptotics as the eigenvalues of the differential equation $[2,3]$. The idea of asymptotic correction is that, before equating the discrete eigenvalues to the eigenvalues of the differential equation, we first add a correction which, in the case of constant potential, makes the discrete eigenvalues exact. For example, consider the corrections to be added to the classical second order centred finite difference approximation of the ith eigenvalue of the Sturm-Liouville problem (1), obtained with uniform mesh length $h$. With the notation $[1,2,3]$

$$
\varepsilon_{r}(i, h)=i^{2}-\frac{12 \sin ^{2}(i h / 2)}{h^{2}\left[3+(1-r) \sin ^{2}(i h / 2)\right]},
$$

the corrections are $\varepsilon_{1}(i, h), \varepsilon_{1}\left(i-\frac{1}{2}, h\right), \varepsilon_{1}\left(i-\frac{1}{2}, h\right)$ and $\varepsilon_{1}(i-1, h)$, when the boundary conditions are, respectively,

$$
\begin{aligned}
& y(0)=y(\pi)=0 \\
& y(0)=y^{\prime}(\pi)=0 \\
& y^{\prime}(0)=y(\pi)=0 \\
& y^{\prime}(0)=y^{\prime}(\pi)=0
\end{aligned}
$$

For the Numerov approximations, the corrections are $\varepsilon_{2}(i, h), \varepsilon_{2}\left(i-\frac{1}{2}, h\right)$, $\varepsilon_{2}\left(i-\frac{1}{2}, h\right)$ and $\varepsilon_{2}(i-1, h)$, respectively, and for the 'linear hat' finite element 
approximations, they are $\varepsilon_{3}(i, h), \varepsilon_{3}\left(\mathfrak{i}-\frac{1}{2}, h\right), \varepsilon_{3}\left(i-\frac{1}{2}, h\right)$ and $\varepsilon_{3}(i-1, h)$, respectively.

Our focus here is on work which postdates my earlier review [3] of work up to about 2004. At that time, most work on the use of asymptotic correction to solve Sturm-Liouville problems had concerned only the symmetric problem [2], though two articles $[2,12]$ also considered one of the problems which use some eigenfunction data ('terminal velocities').

Since then, asymptotic correction has also been used for the two spectra problem. Good results were obtained with a second order method [4], and even better results with either Numerov's method [4] or boundary value methods $[7,20]$. The work described in the previous sentence considered the case where the two sets of boundary conditions are (4) and (5), although inverse problems with quite general boundary conditions can be handled by appropriate modifications [2] of techniques [1] developed for the direct problem. For some boundary conditions, the two spectra problem can be reduced to the symmetric problem [4], but techniques developed specifically for the two spectra problem give better results [4]. A notable limitation of the methods is that, if $\boldsymbol{n}$ eigenvalues of (1) and (4) are used, then the number of eigenvalues of (1) and (5) used has to be either $n$ or $n+1$. Other recent developments include the use of asymptotic correction in methods which allow indefinite mesh refinement [14], and the use of inverse Sturm-Liouville problems in the numerical solution of certain inverse problems for partial differential equations [8].

\section{The Hochstadt-Lieberman problem}

Hochstadt and Lieberman [17] showed that each $q \in \mathrm{L}^{1}(0, \pi)$ is uniquely determined by its value on $(\pi / 2, \pi)$ together with the eigenvalues of $(1)$ and $(2)$. This result has subsequently been generalized in many ways [5]. Gesztesy and Simon [13] considered problems where $\mathbf{q}$ is known on $(\mathbf{c}, \pi)$ for some 
$c \in(0, \pi)$. They showed that, if $0<\mathrm{c}<\pi / 2$, then $\mathrm{q}$ is uniquely determined in $(0, c)$ by a certain fraction of the eigenvalues of $(1)$ and $(2)$, though the number of eigenvalues required is still infinite. If $c>\pi / 2$, then additional data is required to ensure uniqueness on $(0, c)$. We call the case $c=\pi / 2$ the classical Hochstadt-Lieberman problem, and the case of arbitrary $c \in(0, \pi)$ the generalized Hochstadt-Lieberman problem. Since only finitely many eigenvalues will be known in practice, we might expect better accuracy in the case $c<\pi / 2$ when not all eigenvalues are required. Our results (described below) show that achieving this better accuracy is not trivial.

Kammanee and Böckmann [19] used asymptotic correction to solve the classical Hochstadt-Lieberman problem with boundary conditions (4), using a uniform grid and taking as data the $n$ lowest eigenvalues and the values of $q$ at $n$ grid points, so that the known value $q(\pi / 2)$ is not used. Even for the classical problem, we could obtain a slightly finer uniform grid with the same $n$ eigenvalues by using $\pi / 2$ as one of $n+1$ grid points where the known value of $q$ is used. Moreover, in applications [5], the proportion of the interval on which $\mathrm{q}$ is known is usually not exactly half.

Here we consider four cases of the generalized problem. In these cases, which we call 'Problems 1(4), 1(5), 1(6) and 1(7)', the boundary conditions are (4), (5), (6) and (7) respectively. For $r=0,1,2,3$, the data for Problem $1(r+4)$ consists of the values of $q$ at the last $m$ grid points, together with the first $\mathrm{n}$ eigenvalues of (1) for the appropriate boundary conditions. From this data we seek the values of $\mathrm{q}$ at the remaining $\mathrm{n}$ grid points, again using a uniform grid. The same analysis [5] applies to the mirror image problem, where the given values of $\mathbf{q}$ are those at the first $\mathbf{m}$ grid points. The problem studied by Kammanee and Böckmann [19] is the special case $\mathbf{m}=\mathbf{n}$ of Problem 1(4), though their method of solution is slightly different [5]. Before describing our results, we require some notation. Just as in the definition of Problem $1(r+4)$, the values $0,1,2$ and 3 of the parameter $r$ in the definitions of $A_{r}, h_{r}, B_{r}$ and $Q_{r}(\mathbf{q} ; q)$ below represent the values appropriate for boundary conditions (4), (5), (6) and (7), respectively. Our methods iteratively refine a vector, $\mathbf{q}$, whose components, $\mathbf{q}_{1}, \ldots, \mathbf{q}_{\mathfrak{n}}$, approximate the values of $\mathbf{q}$ at the 
first $n$ grid points.

Notation Let $\lambda_{1}^{(\mathrm{r})}<\lambda_{2}^{(\mathrm{r})}<\lambda_{3}^{(\mathrm{r})}<\cdots$ be the eigenvalues of (1) where, in the cases where the parameter $r$ has the values $0,1,2$ and 3 , the boundary conditions are (4), (5), (6) and (7) respectively. Let $A_{0}=\left(a_{i j}\right)$ be the symmetric tri-diagonal $(n+m) \times(n+m)$ Toeplitz matrix with $a_{i i}=2$ and $a_{i, i+1}=-1$. Let $A_{1}$ and $A_{2}$ be the matrices obtained from $A_{0}$ by doubling $a_{n+m, n+m-1}$ and $a_{1,2}$ respectively. Let $A_{3}=A_{1}+A_{2}-A_{0}$, the matrix obtained from $A_{0}$ by doubling both $a_{n+m, n+m-1}$ and $a_{1,2}$. Let $h_{0}=\pi /(n+m+1)$, $h_{1}=h_{2}=\pi /(n+m)$ and $h_{3}=\pi /(n+m-1)$. For $r=0,1,2,3$, let $\Lambda_{i}^{(r)}(\mathbf{q} ; \mathbf{q})$ and $\Lambda_{i}^{(r+4)}(\mathbf{q} ; \mathbf{q})$ be the ith eigenvalues of $h_{r}^{-2} A_{r}+Q_{r}(\mathbf{q} ; \mathbf{q})$ and $h_{r}^{-2} B_{r}^{-1} A_{r}+Q_{r}(\mathbf{q} ; \mathbf{q})$ respectively, where $B_{r}=I-A_{r} / 12$ and $Q_{r}(\mathbf{q} ; \mathbf{q})$ is the $(n+m) \times(n+m)$ diagonal matrix whose ith diagonal element is $q_{i}$ (the ith element of $\mathbf{q}$ ) when $\boldsymbol{i} \leqslant n$, while for $\boldsymbol{i}>\mathrm{n}$ the ith diagonal element of $Q_{r}(q ; q)$ is $q\left(\left(i+s_{r}-1\right) h_{r}\right)$, where $s_{0}=s_{1}=1$ and $s_{2}=s_{3}=0$.

We consider two methods for solving Problem $1(\mathbf{r}+4)$. The first is the classical second order method analogous to one previously used for the symmetric problem [12]. It computes the solution $\mathbf{q}$ of Problem $1(r+4)$, for $r=0,1,2,3$, as the solution of

$$
\mathbf{f}_{\mathrm{r}}(\mathbf{q})=0
$$

where the ith components of the $n$-vectors $\mathbf{f}_{0}(\mathbf{q}), \mathbf{f}_{1}(\mathbf{q}), \mathbf{f}_{2}(\mathbf{q})$ and $\mathbf{f}_{3}(\mathbf{q})$ are $\Lambda_{i}^{(0)}(\mathbf{q} ; q)+\varepsilon_{1}\left(i, h_{0}\right)-\lambda_{i}^{(0)}, \Lambda_{i}^{(1)}(\mathbf{q} ; q)+\varepsilon_{1}\left(i-\frac{1}{2}, h_{1}\right)-\lambda_{i}^{(1)}, \Lambda_{i}^{(2)}(\mathbf{q} ; \mathbf{q})+\varepsilon_{1}(i-$ $\left.\frac{1}{2}, h_{2}\right)-\lambda_{i}^{(2)}$ and $\Lambda_{i}^{(3)}(\mathbf{q} ; \mathbf{q})+\varepsilon_{1}\left(i-1, h_{3}\right)-\lambda_{i}^{(3)}$, respectively. Thus the method seeks a vector $\mathbf{q}$ whose elements are those values of $\mathbf{q}$ at the first $\boldsymbol{n}$ grid points which make the first $\mathrm{n}$ corrected finite difference eigenvalues equal to the (given) Sturm-Liouville eigenvalues.

Our second method uses Numerov's method, with the approximation of the boundary conditions motivated by ideas of Andrew [2,4]. It computes the solutions q of Problems 1(4), 1(5), 1(6) and 1(7) as the solutions of (8) for $r=4,5,6$ and 7 respectively, where the ith components of the $n$-vectors $\mathbf{f}_{4}(\mathbf{q})$, 
$\mathbf{f}_{5}(\mathbf{q}), \mathbf{f}_{6}(\mathbf{q})$ and $\mathbf{f}_{7}(\mathbf{q})$ are $\Lambda_{i}^{(4)}(\mathbf{q} ; \mathbf{q})+\varepsilon_{2}\left(i, h_{0}\right)-\lambda_{i}^{(0)}, \Lambda_{i}^{(5)}(\mathbf{q} ; \mathbf{q})+\varepsilon_{2}\left(i-\frac{1}{2}, h_{1}\right)-$ $\lambda_{i}^{(1)}, \Lambda_{i}^{(6)}(\mathbf{q} ; \mathbf{q})+\varepsilon_{2}\left(i-\frac{1}{2}, h_{2}\right)-\lambda_{i}^{(2)}$ and $\Lambda_{i}^{(7)}(\mathbf{q} ; \mathbf{q})+\varepsilon_{2}\left(i-1, h_{3}\right)-\lambda_{i}^{(3)}$, respectively.

We solve equation (8) using the iteration

$$
\Lambda_{r}^{\prime}(0)\left(\mathbf{q}_{k+1}-\mathbf{q}_{k}\right)=-\mathbf{f}_{r}\left(\mathbf{q}_{k}\right),
$$

where the $(i, j)$ th element of the Jacobian, $\Lambda_{r}^{\prime}(0)$, is $\Lambda_{i, j}^{(r)}(\mathbf{0}, 0)$, the value at $(\mathbf{0}, 0)$ of the partial derivative of $\Lambda_{i}^{(r)}$ with respect to its $j$ th argument. For this iteration to be well defined, we require $\Lambda_{r}^{\prime}(0)$ to be nonsingular. The spectral condition number, $\kappa_{r}(n, m)$, of $\Lambda_{r}^{\prime}(0)$ also plays a critical role in the analysis of the method. The following theorem is an immediate consequence of a stronger, but more complicated, result of Andrew [5].

Theorem 1 Let $\mathrm{n}>1$. For $\mathrm{r}=0,1,2,3, \Lambda_{\mathrm{r}+4}^{\prime}(0)=\Lambda_{\mathrm{r}}^{\prime}(0)$. The $(i, j)$ th elements of $\Lambda_{0}^{\prime}(0)$ and $\Lambda_{1}^{\prime}(0)$ are $2 \sin ^{2}\left(i j h_{0}\right) /(n+m+1)$ and $2 \sin ^{2}((i-$ $\left.\left.\frac{1}{2}\right) j h_{1}\right) /(n+m)$ respectively, while $\Lambda_{2}^{\prime}(0)=M_{1} D$ and $\Lambda_{3}^{\prime}(0)=D M_{2} D$, where the $(i, j)$ th elements of $M_{1}$ and $M_{2}$ are $2 \cos ^{2}\left(\left(i-\frac{1}{2}\right)(j-1) h_{2}\right) /(n+m)$ and $2 \cos ^{2}\left((\mathfrak{i}-1)(j-1) h_{3}\right) /(n+m-1)$ respectively and $D=\operatorname{diag}\left\{\frac{1}{2}, 1, \ldots, 1\right\}$.

If $\mathrm{m}<\mathrm{n}-1$, then, for $\mathrm{r}=0, \ldots, 7, \Lambda_{\mathrm{r}}^{\prime}(0)$ is singular, with rank less than $(\mathrm{n}+\mathrm{m}+2) / 2$.

If $\mathrm{n}-1 \leqslant \mathrm{~m} \leqslant \mathrm{n}+1$, then, for $\mathrm{r}=0, \ldots, 7, \Lambda_{\mathrm{r}}^{\prime}(0)$ is nonsingular and its spectral condition number $\mathrm{K}_{\mathrm{r}}(\mathrm{n}, \mathrm{m})$ satisfies $\mathrm{k}_{0}(\mathrm{n}, \mathrm{m})=\mathrm{O}\left(\mathrm{n}^{1 / 2}\right)$ if $\mathrm{m}=\mathrm{n}$ or $\mathrm{n}-1, \mathrm{\kappa}_{1}(\mathrm{n}, \mathrm{n}+1)=\mathrm{O}\left(\mathrm{n}^{3 / 2}\right)$, while $\mathrm{\kappa}_{\mathrm{r}}(\mathrm{n}, \mathrm{m})=\mathrm{O}(\mathrm{n})$ in all other cases with $\mathrm{r}=0,1,2$ or 3 and $\mathrm{m}=\mathrm{n}-1, \mathrm{n}$ or $\mathrm{n}+1$.

Also, for all $\mathrm{n}, \mathrm{m} \in \mathbb{N}$ such that $\Lambda^{\prime}(0)$ is nonsingular (even if $\mathrm{m}>\mathrm{n}+1$ ), and for all $\mathrm{q}^{*} \in \mathbb{R}$ and all $\mathrm{p} \geqslant 1$, there exist constants $\mathrm{c}_{\mathrm{p}}(\mathrm{n}, \mathrm{m})$ and $\mathrm{c}(\mathrm{n}, \mathrm{m})$ such that, if (i) $\left\|\mathrm{q}-\mathrm{q}^{*}\right\|_{\mathrm{p}}<\mathrm{c}_{\mathrm{p}}(\mathrm{n}, \mathrm{m})$, and (ii) for $\mathrm{i}=1, \ldots, \mathrm{n}, \mid \mathrm{q}_{\mathrm{i} 0}-$ $\mathrm{q}\left(\left(\boldsymbol{i}+s_{\mathrm{r}}-1\right) \mathrm{h}_{\mathrm{r}}\right) \mid<\mathrm{c}(\mathrm{n}, \mathrm{m})$, where $\mathrm{q}_{\mathrm{i} 0}$ is the $i$ th component of the initial approximation $\mathbf{q}_{0}$, then, for $\mathrm{r}=0,1,2,3$, the iteration (9) converges to a solution of (8), and this solution is locally unique. This conclusion is also 
true for $\mathrm{r}=4,5,6,7$, provided the eigenvalues of $\mathrm{h}^{-2} \mathrm{~B}_{\mathrm{r}-4}^{-1} \mathrm{~A}_{\mathrm{r}-4}+\mathrm{Q}_{\mathrm{r}-4}\left(\mathbf{q}_{\mathrm{k}} ; \mathrm{q}\right)$ are simple for all $\mathrm{k}$.

The restriction to simple eigenvalues imposed in the last sentence of Theorem 1 is not significant in practice [5]. The real restriction is the size of the constants $c_{p}(n, m)$ and $c(n, m)$, which rapidly become small as $k_{r}(n, m)$ increases [5]. Theorem 1 shows that, for boundary conditions (4) and (5), $\kappa_{r}(n, m)$ increases by a factor $O\left(n^{1 / 2}\right)$ when $m$ increases from $n$ to $n+1$, but, when $m>n+1$, it gives no information on $\kappa_{r}(n, m)$, or even on whether $\Lambda_{r}^{\prime}(0)$ is singular. However, numerical results [5] show that $\Lambda_{r}^{\prime}(0)$ remains nonsingular for $m>$ $n+1$, but that $\kappa_{r}(n, m)$ increases quite rapidly as $m$ is increased beyond $n+1$, especially for Problem 1(5). This increase in $\kappa_{r}(n, m)$ substantially reduces the size of the constants $c_{p}(n, m)$ and $c(n, m)$ in Theorem 1 , and also increases the sensitivity of the computed solution to errors in the eigenvalue data [5]. This limits the range of values of $(m-\mathfrak{n})$ for which the method is effective, though the limitation is not as severe as for the two spectra problem [4].

Nevertheless the corrected Numerov method performed well for a modest range of $m-n$. For a given (small) value of $m-n$ and for $q \in C^{1}$, it gave $\mathrm{O}\left(\mathrm{h}_{\mathrm{r}}^{3}\right)$ convergence to the true value of $\mathrm{q}$ as mesh length $h_{r} \rightarrow 0$ except near the boundary, where convergence was $\mathrm{O}\left(h_{r}\right)$. For $\mathrm{q}(x)=|2-x|$, convergence was also $\mathrm{O}\left(h_{r}\right)$ near $(\pi-2)$, the mirror image point of the discontinuity of $\mathrm{q}^{\prime}$ at 2 . Convergence near 2 for the problem with $\mathrm{q}$ given on $(0, \pi / 2)$ was also $\mathrm{O}\left(h_{\mathrm{r}}\right)$. At other points away from the boundaries, convergence, though less regular than for $q \in C^{1}$, was still better than $\mathrm{O}\left(\mathrm{h}_{\mathrm{r}}^{2}\right)[5]$.

Why does a discontinuity of $\mathbf{q}^{\prime}$ at 2 affect the convergence of our method not only at 2 but also at the mirror image point $(\pi-2)$ ? A possible explanation is the importance of Fourier coefficients in the asymptotic expansion of the eigenvalues $[2,4,5,12]$. For example, it is known [5] that if $\mathbf{q} \in \mathrm{L}^{2}(0, \pi)$ then $\lambda_{i}^{(0)}=i^{2}+\bar{q}-\pi^{-1} \int_{0}^{\pi} q(t) \cos (2 i t) d t+\alpha_{i} / i$, where $\bar{q}$ is the mean value of $q$ and $\left\{\alpha_{i}\right\}_{i=1}^{\infty} \in \ell^{2}$. The even Fourier cosine coefficients give information about $(q+\widehat{q}) / 2$ (the part of $q$ that is even about $\pi / 2$ ) rather than the odd part, $(q-\widehat{q}) / 2$. Since generally $\alpha_{i} \neq 0$, not all the information in the eigenvalues 
concerns these Fourier coefficients, but dominance of the Fourier term could be expected to lead to some symmetry in the errors.

Table 1 shows another symmetry result, which was apparent in all our numerical results, especially those for smoother $\mathrm{q}$. The errors in our computed results normally changed significantly when $\mathbf{q}$ in (1) was replaced by $\mathbf{q}+\delta \mathbf{q}$, even when $\delta q$ was quite small, but when $q$ was replaced by its mirror image $\widehat{\mathrm{q}}$, the change in the error obtained by Numerov's method was very small, although none of our test problems involved symmetric q. Table 1 compares the errors for Problem 1(4) when $\mathrm{q}(x)=x^{2}(\pi-x) / 2$, so that $\widehat{\mathrm{q}}(x)=x(\pi-x)^{2} / 2$. Similar symmetry effects appeared in the results obtained by the second order method, but the effect was much less clear than with Numerov's method. Note that, because of the high accuracy of our results, the errors in Table 1 are multiplied by $10^{3}$ for ease of tabulation.

Table 1 also illustrates another feature of our Numerov results for $\mathrm{C}^{1}$ functions. The errors at successive grid points generally alternated in sign and decreased monotonically in magnitude away from the boundary. (The very few exceptions occur at points where the error was less than about $10^{-7}$ and can be explained by tiny errors in the eigenvalue data.)

For both the Hochstadt-Lieberman problem and the two spectra problem we have similar limitations on the relative sizes of the two data sets used. The extent to which these limitations can be overcome is an open question. Our method for the two spectra problem [4] requires that exactly $n$ or $n+1$ eigenvalues of (1) and (5) must be used when $\boldsymbol{n}$ eigenvalues of (1) and (4) are used. This is related to the fact that, in a sense, the corresponding uniqueness theorem requires that half of the information be provided by each of (4) and (5), though adding a finite number of elements to an infinite set does not change its cardinality. A method [19] suggested for supplementing the eigenvalue set for the Hochstadt-Lieberman problem may be more useful for the two spectra problem. This is because it is normally the lowest eigenvalues that are known, and the method uses interpolation when missing eigenvalues are lower than some known ones (as is the case for the two spectra problem), 
TABLE 1: $10^{3} \times($ Error at $j h)$ in the Numerov results when $n=30$ and $\mathrm{q}(x)=x^{2}(\pi-x) / 2$.

\begin{tabular}{rrrrrrr}
\hline $\mathrm{m}-\mathrm{n}$ & \multicolumn{2}{c}{-1} & \multicolumn{2}{c}{2} & \multicolumn{2}{c}{$\mathrm{q}$} \\
\hline $\mathrm{j}$ & $\mathrm{q}$ & $\widehat{\mathrm{q}}$ & $\mathrm{q}$ & $\widehat{\mathrm{q}}$ & $\mathrm{q}$ & $\widehat{\mathrm{q}}$ \\
\hline 1 & -4.915 & -4.917 & -4.823 & -4.825 & -4.673 & -4.675 \\
2 & 1.758 & 1.762 & 1.718 & 1.721 & 1.666 & 1.670 \\
3 & -0.874 & -0.875 & -0.848 & -0.849 & -0.824 & -0.825 \\
4 & 0.516 & 0.517 & 0.496 & 0.498 & 0.483 & 0.486 \\
5 & -0.343 & -0.343 & -0.325 & -0.326 & -0.318 & -0.319 \\
10 & 0.095 & 0.096 & 0.081 & 0.081 & 0.081 & 0.081 \\
15 & -0.048 & -0.048 & -0.034 & -0.034 & -0.035 & -0.036 \\
20 & 0.034 & 0.032 & 0.017 & 0.017 & 0.018 & 0.018 \\
25 & -0.026 & -0.025 & -0.006 & -0.007 & -0.008 & -0.008 \\
30 & 0.001 & 0.001 & 0.001 & 0.001 & 0.002 & 0.002 \\
\hline
\end{tabular}

but requires the riskier process of extrapolation when the known eigenvalues are all less than the missing ones.

The requirement $m \geqslant n-1$ in Theorem 1 may also be related to the uniqueness result [17]. I plan to consider elsewhere the case $m<n-1$ with not all known eigenvalues coming from the same boundary conditions. However, since the problem should be easier to solve when we have more prior information on $q$, the problems associated with larger values of $m-n$ may be more tractable. This suggests some ideas for future research. The traditional choice of uniform mesh was made because it gives a closed form expression for the correction, but asymptotic correction was also successful when no closed form expression is available $[14,20]$. It is possible a suitable choice of mesh could reduce the growth of $\kappa_{r}(n, m)$ [5]. Since the proof [5] of convergence in Theorem 1 does not require the correction be calculated using constant q, it may also be useful to calculate the correction as the error in the finite difference estimates obtained with some other choice $\tilde{\mathbf{q}}$ of $\mathbf{q}$, using the known values of $\mathbf{q}$ on $(\mathbf{c}, \pi)$ [5]. Results of Rafler and Böckmann [29] 
for another method, suggest that a non-constant $\tilde{q}$ may be especially useful when $\mathrm{q}$ has jump discontinuities. If initial results indicate that $\mathbf{q}$ has jump discontinuities, the choice of $\tilde{\mathbf{q}}$ could be refined iteratively so that it has similar discontinuities.

\section{References}

[1] A. L. Andrew. Asymptotic correction of more Sturm-Liouville eigenvalue estimates. BIT, 43:485-503, 2003. MR 2004m:65104. doi:10.1023/B:BITN.0000007052.66222.6d C290, C291

[2] A. L. Andrew. Numerov's method for inverse Sturm-Liouville problems. Inverse Problems, 21:223-238, 2005. MR 2006d:65076. doi:10.1088/0266-5611/21/1/014 C288, C289, C290, C291, C293, C295

[3] A. L. Andrew. Asymptotic correction and inverse eigenvalue problems: an overview. ANZIAM J., 46(E):C1-C14, 2005. Zbl 1078.65558. http://anziamj . austms.org.au/V46/CTAC2004/Andr C290, C291

[4] A. L. Andrew. Computing Sturm-Liouville eigenvalues from two spectra. Inverse Problems 22:2069-2081, 2006. MR 2007i:34013. doi:10.1088/0266-5611/22/6/010 C289, C290, C291, C293, C295, C296

[5] A. L. Andrew. Finite difference methods for half inverse Sturm-Liouville problems. Appl. Math. Comp., (to appear). doi:10.1016/j.amc.2011.05.085 C289, C290, C291, C292, C294, C295, C297

[6] A. L. Andrew and J. W. Paine. Correction of Numerov's eigenvalue estimates. Numer. Math., 47:289-300, 1985. MR 86j:65101. doi:10.1007/BF01389712 C289 
[7] C. Böckmann and A. Kammanee. Broyden method for inverse non-symmetric Sturm-Liouville problems. BIT, to appear. doi:10.1007/s10543-011-0317-5 C291

[8] A. Boumenir and V. K. Tuan. An inverse problem for the heat equation. Proc. Amer. Math. Soc. 138:3911-3921, 2010. Zbl 1202.35344. doi:10.1090/S0002-9939-2010-10297-6 C291

[9] K. Chadan, D. Colton, L. Päivärinta and W. Rundell. An introduction to inverse scattering and inverse spectral problems. SIAM, 1993. C289

[10] M. T. Chu. Inverse eigenvalue problems. SIAM Rev., 40:1-39, 1998. http://www.siam.org/journals/sirev/40-1/30398.html C288

[11] C. R. Dun and R. S. Anderssen. Algebraic correction methods for inverse Sturm-Liouville problems. In D. Stewart, H. Gardner and D. Singleton, editors, Computational Techniques and Applications: CTAC93, pages 202-210. World Scientific, 1994. C289

[12] R. H. Fabiano, R. Knobel and B. D. Lowe. A finite difference algorithm for an inverse Sturm-Liouville problem. IMA J. Numer. Anal. 15:75-88, 1995. doi:10.1093/imanum/15.1.75 C291, C293, C295

[13] F. Gesztesy and B. Simon. Inverse spectral analysis with partial information on the potential II The case of discrete spectrum. Trans. Amer. Math. Soc. 352:2765-2787, 2000. MR 2000j:34019. doi:10.1090/S0002-9947-99-02544-1 C291

[14] P. Ghelardoni and C. Magherini. BVMs for computing Sturm-Liouville symmetric potentials. Appl. Math. Comp. 217:3032-3045, 2010. doi:10.1016/j.amc.2010.08.036 C289, C291, C297

[15] G. M. L. Gladwell 2004 Inverse Problems in Vibration 2nd edn. Dordrecht: Kluwer Academic Publishers, 2004. MR 2005g:74059. C289 
[16] O. H. Hald and J. R. McLaughlin. Solutions of inverse nodal problems. Inverse Problems 5:307-347, 1989. MR 90c:34015. doi:10.1088/0266-5611/5/3/008 C289

[17] H. Hochstadt and B. Lieberman. An inverse Sturm-Liouville problem with mixed given data. SIAM J. Appl. Math. 34:676-680, 1978. MR 57\#10077. doi:10.1137/0134054 C291, C297

[18] M. Ignatiev and V. Yurko. Numerical methods for solving inverse Sturm-Liouville problems. Result. Math. 52:63-74, 2008. MR 2009c:65160. doi:10.1007/s00025-007-0276-y C289

[19] A. Kammanee and C. Böckmann. Determination of partially known Sturm-Liouville potentials. Appl. Math. Comp. 204:928-937, 2008. doi:10.1016/j.amc.2008.08.001 C292, C296

[20] A. Kammanee and C. Böckmann. Boundary value method for inverse Sturm-Liouville problems. Appl. Math. Comp. 214:342-352, 2009. MR 2010g:65090. doi:10.1016/j.amc.2009.04.002 C291, C297

[21] B. D. Lowe, M. Pilant and W. Rundell. The recovery of potentials from finite spectral data. SIAM J. Math. Anal. 23:482-504, 1992. MR 93f:34024. C288

[22] J. T. Marti. Small potential corrections for the discrete eigenvalues of the Sturm-Liouville problem. Numer. Math. 57:51-62, 1990. doi:10.1007/BF01386396 C290

[23] M. Neher. Enclosing solutions of an inverse Sturm-Liouville problem with finite data. Computing 53:379-395, 1994. MR 95i:65111. doi:10.1007/BF02307388 C288, C289

[24] J. Paine. A numerical method for the inverse Sturm-Liouville problem. SIAM J. Sci. Stat. Comput. 5:149-156, 1984. MR 85c:65097. doi:10.1137/0905011 C290 
[25] J. Paine. Regularization applied to the recovery of piecewise constant Sturm-Liouville potentials Proc. CMA, Austral. Nat. Univ. 17:106-116, 1988. MR 90d:65152. C289

[26] J. W. Paine, F. R. de Hoog and R. S. Anderssen. On the correction of finite difference eigenvalue approximations. Computing 26:123-139, 1981. doi:10.1007/BF02241779 C289

[27] J. Pöschel and E. Trubowitz. Inverse spectral theory. Academic Press, 1987. MR 89b:34061. C289

[28] J. D. Pryce. Numerical solution of Sturm-Liouville problems. Oxford University Press, 1993. MR 95h:65056. C290

[29] M. Rafler and C. Böckmann. Reconstructive method for inverse Sturm-Liouville problems with discontinuous potentials. Inverse Problems 23:933-946, 2007. doi:10.1088/0266-5611/23/3/006 C289, C297

[30] N. Röhrl. A least-squares functional for solving inverse Sturm-Liouville problems Inverse Problems 21: 2009-2017, 2005. MR 2006h:34024. doi:10.1088/0266-5611/21/6/013 C289

[31] W. Rundell and P. E. Sacks. Reconstruction techniques for classical Sturm-Liouville problems. Math. Comp. 58:161-183, 1992. doi:10.1090/S0025-5718-1992-1106979-0 C289

[32] V. V. Ternovskii and M. M. Khapaev. Reconstruction of potentials in the Sturm-Liouville inverse problem by the variational method. Dokl. Math. 73:107-111, 2006. doi:10.1134/S1064562406010297 C289 


\section{Author address}

1. Alan L. Andrew, Dept. Mathematics, La Trobe University, Victoria 3086, Australia.

mailto:a.andrew@latrobe.edu.au 